\title{
Establishment and Characterization of a Stromal Cell Line Derived From a Patient With Thoracic Endometriosis
}

Reproductive Sciences

1627-1636

(C) The Author(s) 2019

DOI: $10.1007 / s 43032-020-00193-8$

\author{
J. Gogusev, MD, PhD', Y. Lepelletier, $\mathrm{PhD}^{2}$, \\ L. El Khattabi, MD, PhD ${ }^{3}$, M. Grigoroiu ${ }^{4}$, and P. Validire ${ }^{5}$
}

\begin{abstract}
Thoracic endometriosis (TE) syndrome is a clinical condition known as an extrapelvic form of endometriosis with the presence of functioning endometrial tissue involving lung parenchyma, pleura, chest wall, or diaphragm. In an effort to obtain an endometriosis ex vivo model, we established the spontaneously growing TH-EMI cell line from endometriotic implants in lung parenchyma from a woman with TE. Maintained in long-term culture, the cells grew as large mesenchymal-like cells with a doubling time between 5 and 6 days. Treatment with medroxyprogesterone acetate $\left(10^{-7} \mathrm{~mol} / \mathrm{L}\right)$ inhibited the TH-EMI cells growth and induced morphological changes to an epithelial-like cells. Strong expression of the nuclear estrogen receptors, progesterone receptors, and erytropoietin receptors were found in both the pulmonary implant and the $\mathrm{TH}$-EMI cells by immunohistochemical analysis. Consistent immunoreactivity of TH-EMI cells for CD9, CDI3, CD73, CD90, CDI05, and CDI57 was revealed by flow cytometry. Likewise, the embryonic markers, SRY-box 2 (SOX-2) and the Nanog molecules, were detected in $76 \%$ and $52 \%$ of the cells, while fetal hemoglobin and $\alpha$-globin were detected in $76 \%$ and $65 \%$ of TH-EMI cells, respectively. By RHG banding, normal metaphases were observed, while the microarray chromosomal analysis showed gains of DNA sequences located on the segments 8p23.I, I I P 5.5, and I2p I I.23. The described in vitro cellular model can serve as a useful tool to study the pathogenesis of endometriosis and to improve the knowledge of molecular mechanisms controlling the endometriotic cell dissemination potential.
\end{abstract}

\section{Keywords}

thoracic endometriosis (TE) syndrome, cell line, karyotype, microarray chromosome analysis (MCA)

\section{Introduction}

Endometriosis is a hormone-dependent disease characterized by the presence of endometrial-like glands in ectopic localization, affecting between $5 \%$ and $10 \%$ of women in childbearing age. A variety of extra-pelvic endometriosis is the thoracic endometriosis (TE) syndrome that can involve the airways, pleura, lung parenchyma, and diaphragm. ${ }^{1-3}$ Clinically, TE comprises several entities such as catamenial hemoptysis and catamenial pneumothorax and shows the presence of lung and/or diaphragmatic tissue lesions. ${ }^{4}$ Today, the prevalence of TE remains unknown due to the lack of well-designed studies conducted on larger cohorts of patients. The most extensive clinical survey included 110 patients with TE and showed that the highest incidence for pelvic endometriosis ranges between 24 and 29 years, whereas the peak occurrence for TE syndrome is approximately 5 years later. ${ }^{5}$ One of the theories attempting to explain the pathogenesis of TE syndrome is the concept of lymphatic or hematogenic dissemination of pelvic endometriosis to lung or pleural locations. ${ }^{2,6,7}$ Likewise, the lymphatic spread theory has been proposed to explain the occurrence of endometriosis in other distant locations. ${ }^{8}$ The next theory suggests that catamenial pneumothorax occurs after migration of implants from the pelvic cavity through diaphragmatic defects by leakage of air occurring when the endometriotic tissue breaks down during menstruation. ${ }^{1,9}$ Alternatively, committed stromal mesenchymal cells issued from the endometrium or from the bone marrow may as

\footnotetext{
' Cochin Institute, Inserm UMR 1016, CNRS 8104, Université Paris Descartes, Paris, France

${ }^{2}$ Imagine Institute, Inserm UMR II63, CNRS ERL 8254, Université Paris Descartes-Sorbonne Paris Cité, Paris, France.

${ }^{3}$ Service de Cytogénétique, AP-HP, Hôpital Cochin, Inserm UI016, CNRS 8104, Université Paris Descartes, Paris, France

${ }^{4}$ Service de Chirurgie Thoracique, Institut Mutualiste Montsouris, Paris, France

${ }^{5}$ Service d'Anatomie Pathologique, Institut Mutualiste Montsouris, Paris, France
}

\section{Corresponding Author:}

J. Gogusev, Cochin Institute, Inserm UMR 1016, CNRS 8104, Université Paris Descartes, 24 rue du faubourg St Jacques, Paris 75014, France.

Email: jean.gogusev@gmail.com 
well be transported via the lymphatic or vascular pathways to generate lesions in different ectopic sites including lung parenchyma. ${ }^{10,11}$ A further possibility might be development of TE lesions as an independent pathological process generated from a confined abnormal clone of pluripotential cells able to transform into endometrial tissue. ${ }^{12}$

In the present report, we describe the establishment of THEM1 cell line derived from subpleural implants located on the apical pole of the right lung and on the diaphragm in patient with TE. The cell line grew spontaneously and exhibited particular proliferative, phenotypic, and molecular cytogenetic features suggestive of a mesenchymal stromal cell-like lineage involved in the pathogenesis of TE.

\section{Patients and Methods}

\section{Patient and Endometriotic Tissue Culture}

A 32-year-old patient was admitted at the Department of Thoracic Surgery (Institut Mutualiste Montsouris, Paris, France) for complaint of episodes of right pulmonary pain and hemoptysis. The clinical history indicated that 2 years ago, this patient was treated with GnRH agonists for pelvic endometriosis. The blood picture and the tuberculosis workup were negative, and she had no history of smoking.

On admission, the chest radiography showed right-sided pneumothorax with significant pleural effusion. The magnetic resonance imaging confirmed the presence of small nodules located on the apical pole of the right lung. A surgical thoracotomy was performed where multiple hemorrhagic small nodular implants were observed on the pleural surface spreading into the apical pole of the right lung parenchyma. Additional, soft red-browncolored lesion was observed on the thoracic tendinous diaphragmatic surface surrounded by hemorrhagic spots and islands of vascular networks. The apical pulmonary lesion $(4.5 \times 1 \times 0.5$ $\mathrm{cm}^{3}$ in diameter) was excised, and in parallel, biopsies of the pleural nodules were performed. A second red-colored diaphragmatic lesion $\left(4.5 \times 3 \times 0.5 \mathrm{~cm}^{3}\right)$ located at the tendinous part of the right diaphragm was also resected, followed by intercostal pleural drainage that achieved a good postsurgical procedure recovery. Forth-generation progestogen therapy was given with good clinical response. Medication was discontinued 4 months later, and after 24 months of follow-up, the patient remained clinically normal and had no episodes of hemoptysis.

\section{Tissue and Cell Culture}

A small tissue fragment $\left(1 \times 0.5 \mathrm{~cm}^{2}\right)$ was dissected from the large pulmonary tissue fragment and given to us to generate the cell culture. The remaining fragment and the lesion from the tendinous part of the right diaphragm were fixed in buffered formalin and processed for routine histopathology. Serial tissue sections $(4 \mu \mathrm{m})$ were performed for establishment the histopathological diagnosis and immunohistochemical parameters. The institutional ethics committee approved this investigation, and patient consent was obtained before tissue collection.
Cell culture was performed following the standard tissue culture procedures. Briefly, the tissue fragment was mechanically dissociated, incubated in $0.2 \%(2 \mathrm{mg} / \mathrm{mL})$ of Collagenase II (Sigma-Aldrich Chimie, Saint Quentin Fallavier, France) during 2 hours at $37^{\circ} \mathrm{C}$, and the cell suspension was sieved through a sieve of $0.3 \mathrm{mmol} / \mathrm{L}$. The cell suspension containing small cellular aggregates was then allowed to attach to the culture flask and incubated in Dulbecco modified Eagle medium supplemented with $10 \%$ of fetal calf serum (FCS), L-glutamine, and antibiotics (Invitrogen-Thermo Fisher, Cergy-Pontoise, France). After several days of growing, stromal-like cells admixed with large epithelial cells, adherent macrophages, and fibroblast-like cells were observed on phase-contrast microscopy. After 1 or 2 passages in the utilized culture medium, the epithelial and macrophage cells died and were overgrown by mesenchymal cells. After 5 to 6 weeks of culture, adherent cell populations including polygonal and spindle-shaped fibroblast-like cells started to proliferate more rapidly, and since then, the cell line named TH-EM1 was serially passaged at a density of $2 \times 10^{5}$ cells $/ \mathrm{mL}$ for more than 45 passages.

\section{Measurement of Cell Proliferation}

The cell proliferation assay was performed by plating the cells in triplicates at a concentration of 1000 cells per $\mathrm{cm}^{2}$ in a 25 $\mathrm{cm}^{2}$ culture flasks. Exponentially growing cells were plated in flasks containing $5 \mathrm{~mL}$ of culture medium at a concentration of $1 \times 10^{5}$ cells $/ \mathrm{mL}$. The cells were cultured for 7 days and at $24-$ hour intervals enzymatically detached and resuspended in $5 \mathrm{~mL}$ of medium. In a separate experiment, TH-EM1 cells were treated with medroxyprogesterone acetate (MPA) alone at a concentration of $10^{-7} \mathrm{~mol} / \mathrm{L}$, (Sigma Aldrich) to compare the cell growth kinetics after stimulation. The cell number in triplicate (0.5-mL aliquots of each sample) was determined using a Coulter Multisizer II (Beckman Coulter, France). The cell population doubling time (PDT) was determined at the time of exponential growth (log phase), which was between 48 and 96 hours after initial plating, using the formula: PDT $=(\ln (2)$ $\times t) / \ln (\mathrm{Nt} / \mathrm{No}$ ), where $\mathrm{t}$ is time interval (48 hours), $\mathrm{Nt}$ is number of cells at 96 hours, and No is number of cells at 48 hours.

\section{Immunohistochemistry}

Serial sections of the pulmonary and diaphragmatic implant tissues were deparaffinized, dehydrated in ethanols, and heated at $65^{\circ} \mathrm{C}$ for antigen retrieval before treatment with the specific antibodies (Table 1). For most of the antibodies, the standard indirect immunoperoxidase method was employed. Immunocytochemical analysis of TH-EM1 cell line was performed on cells detached from the tissue culture flaks and prepared as cytospins on slides; to note, this cytospinning procedure results in round cell morphology, different from that of endometrial mesenchymal cells prepared in situ. Thereafter, the cells were washed, fixed in cold acetone, rinsed in phosphate-buffered saline (PBS), and incubated with specific primary antibodies. The immunostaining reaction was evaluated with a panel of monoclonal 
Table I. Immunohistochemistry/cytochemistry of the Original Thoracic Endometriotic Lesion and TH-EMI Cell Line.

\begin{tabular}{|c|c|c|c|c|}
\hline Antibody Used & Specificity and/or Cell Type & Source & TE Lesion Staining & TE-EMI, \% ${ }^{\mathrm{a}}$ \\
\hline Vimentin (cl.V9) & Mesenchymal cells & Dako-Agilent & Moderate & $95 \%$ \\
\hline Cytokeratin (AEI/AE3) & Epithelial cells & Dako-Agilent & Weak/moderate & $2 \%-3 \%$ \\
\hline WTI & Wilms tumor protein I & Dako-Agilent & Moderate & $65 \%$ \\
\hline CD9 & $\begin{array}{l}\text { Leukocyte, platelets, monocytes, neurons, } \\
\text { glial cells, epithelial cells }\end{array}$ & Dako-Agilent & ND & $50 \%$ \\
\hline PR & Progesterone receptor & Dako-Agilent & Strong & $? 87 \%$ \\
\hline ER & Estrogen receptor & Dako-Agilent & Strong & $? 65 \%$ \\
\hline HLA-DR & B cells, monocytes, macrophages, dendritic cells & Dako-Agilent & Weak & $0 \%$ \\
\hline PAX8 & Ovarian epithelial cells & Abcam & Moderate in stromal clusters & $54 \%$ \\
\hline Fet-Hb & Fetal hemoglobin & Santa Cruz & Weak & $67 \%$ \\
\hline EpoR & Bone marrow cells, stromal cells & Thermo-Fisher & Moderate & $78 \%$ \\
\hline
\end{tabular}

Abbreviations: EpoR, erytropoietin receptor; ER, estrogen receptor; Fet-Hb, fetal hemoglobin; ND, not done; PR, progesterone receptor; SOX-2, SRY-box 2; TE, Thoracic endometriosis.

${ }^{a}$ aPercentage of immunoreactive TH-EMI cells.

antibodies (mAbs) or polyclonal antibodies at appropriate dilution listed in Table 1 by counting at least 200 cells. The antibodies were revealed using rabbit anti-mouse or rabbit anti-goat immunoglobulin $\mathrm{G}(\mathrm{IgG})$ peroxidase-conjugated antibodies (LSAB2 system; Dako, Glostrup, Denmark) according to the manufacturer's instructions. The control slides for both tissue sections and cells were incubated with normal mouse serum to replace the specific antibodies. For the immunohistochemical staining evaluation, the number of immunostained cells was counted and averaged in 5 different fields with $40 \times$ objective. Immunoreactivity was evaluated semiquantitatively as follows: strong staining (presence of more than $65 \%$ of stained cells), moderate staining (more than $30 \%$ of stained cells), weak staining ( $10 \%$ of labeled cells), and negative staining (not significantly greater than that of the control slides). For cultured cells, at least 2 slides treated with the same antibody were scanned from one end to the other of the cytospin spot until 500 cells were reached, and the percentage was calculated. All evaluations were performed by consensus by the 2 authors.

\section{Flow Cytometry}

Untreated proliferating TH-EM1 cells $\left(2 \times 10^{5}\right.$ cells $)$ were harvested and washed with $1 \times \mathrm{PBS} \mathrm{Ca}{ }^{2+} / \mathrm{Mg}^{2+}$ buffer containing $2 \%$ FCS. Then, cells were directly stained with the appropriate antibody using PBS $(1 \times)$ buffer supplemented with $2 \%$ FCS for detection of cell membrane antigens. For determination of intracytoplasmic or nuclear antigens, the cells were fixed in $4 \%$ paraformaldehyde and permeabilized with $1 \%$ saponin during 15 minutes at $4{ }^{\circ} \mathrm{C}$. The unlabeled primary antibodies were revealed by incubation with goat anti-mouse IgG-PE (Jackson ImmunoResearch, Bar Harbor, ME) secondary antibody at $4^{\circ}$ for 15 minutes. The mABs directly labeled by phycoerythrin were anti-fetal hemoglobin (Fet-Hb) and CD31 (Becton Dickinson, France), anti-CD157, and CD105 from e-BiosciencesThermo Fisher. The mABs used and labeled by fluorescein isothiocyanate were CD9, CD10, CD44, CD13, HLA-DR (Becton Dickinson), and anti-CD73 from e-Biosciences. The allophycocyanin (APC)-labeled mABs anti-CD90 and antiCD117 were obtained from Becton Dickinson. As control, the cells were stained using unrelated antibody labeled with the appropriate fluorescent dye (Becton Dickinson). The analysis of embryonal marker expression was performed after incubation of the TH-EM1 cells with unlabeled antiSOX-2 and anti-Nanog primary antibodies (e-Biosciences) that were revealed by the appropriate PE-labeled secondary antibody (e-Biosciences). The Oct $/ 3 / 4$ molecule was revealed using the directly labeled (phycoerythrin (PE) Oct $/ 3 / 4$ antibody, e-Biosciences). The stained cells were analyzed on a fluorescence-activated cell sorter, FACS Calibur flow cytometer (Becton Dickinson Co, Mountain View, California), and data analysis was performed with Flowjo software version 10-2.

\section{Conventional Cytogenetics and Microarray Chromosome Analysis}

The TH-EM1 cell metaphases from both early (fourth passage) and the 15th and 43rd passages were R-banded karyotype (RGH) banded according to standard procedures. Between 20 and 30 metaphases were counted, and 15 of them were photographed and analyzed.

For microarray chromosome analysis, DNA was isolated from the fourth passage of TH-EM1 cells and profiled for copy number using an oligonucleotide DNA microarray platform (Sure Print G3 Human CGH microarray $8 \times 60 \mathrm{~K}$; Agilent 
Technologies, California). For cohybridization against the tested DNA, an in-house reference DNA was used isolated from multiple normal individuals. The DNAs were digested, labeled, and hybridized according to manufacturer's protocol (Agilent Technologies). Data were analyzed using the algorithm ADM-2 (Agilent). Genomic amplifications and deletions were considered when at least 5 oligoprobes presented values of $\log 2$ above or below 0.25 , respectively.

\section{Results}

\section{Histopathology of the Lung Endometriotic Implant}

Hematoxylin and eosin-stained paraffin section of the implanted tissue in the right lung showed presence of rare morphologically altered glandular structures lined by cuboidal cells, surrounded by round-shaped, stromal-like cells and small hemorrhagic areas (Figure 1A). Stained sections from the pulmonary implant and from the diaphragmatic nodular tissue showed several isolated clusters composed of elongated stromal cells with round and ovoid nuclei were readily observed (Figure 1B). Neither cellular atypia nor necrosis was seen. Foci of mesothelial hyperplasia were also noted at the periphery of the analyzed lung fragment (not shown).

\section{Growth Characteristics of TH-EMI Cell Line}

On phase-contrast microscopy, the primary culture appeared as adherent cell aggregates growing as an admixture of spindle-shaped polygonal cells. The admixed cells proliferated mostly as a monolayer, often forming areas of multilayered growth. From the early passages (second to fourth), the cells appeared rather polygonal and irregularly shaped with ovoid nuclei, abundant cytoplasm, and showed cytoplasmic extensions. Between the passages 5 and 6 , the cells underwent morphological changes appearing mainly as large spindle-type cells that begun to grow more rapidly and thereafter could be serially cultured at a dilution of 1:2 every week. From that time period, this new cell line designated TH-EM1 has been stably maintained for more than 45 passages in vitro, and the PDT of 5 to 6 days had not varied over 2 years in culture (Figure 2A). The proliferative potential of the TH-EM1 cells in presence of $10 \%$ FCS remained unchanged during the passages, whereas treatment with MPA $\left(10^{-7}\right)$ inhibited the TH-EM1 cell proliferation and induced appearance of large epithelioid-like cells (Figure 2B). The evaluated doubling time, from the time course experiments, was 5 to 6 days approximately with a mean PDT of 96 hours in the exponential growth phase (Figure 2C).

\section{Immunohistochemical Findings}

The immunohistochemical profile of the original implant in lung in comparison to the TH-EM1 cells is shown in Figure 3 and Table 1. The staining for the epithelial marker using antipan cytokeratin antibodies showed cytoplasmic immunolabeling restricted to the gland lining epithelial cells (Figure 3A).

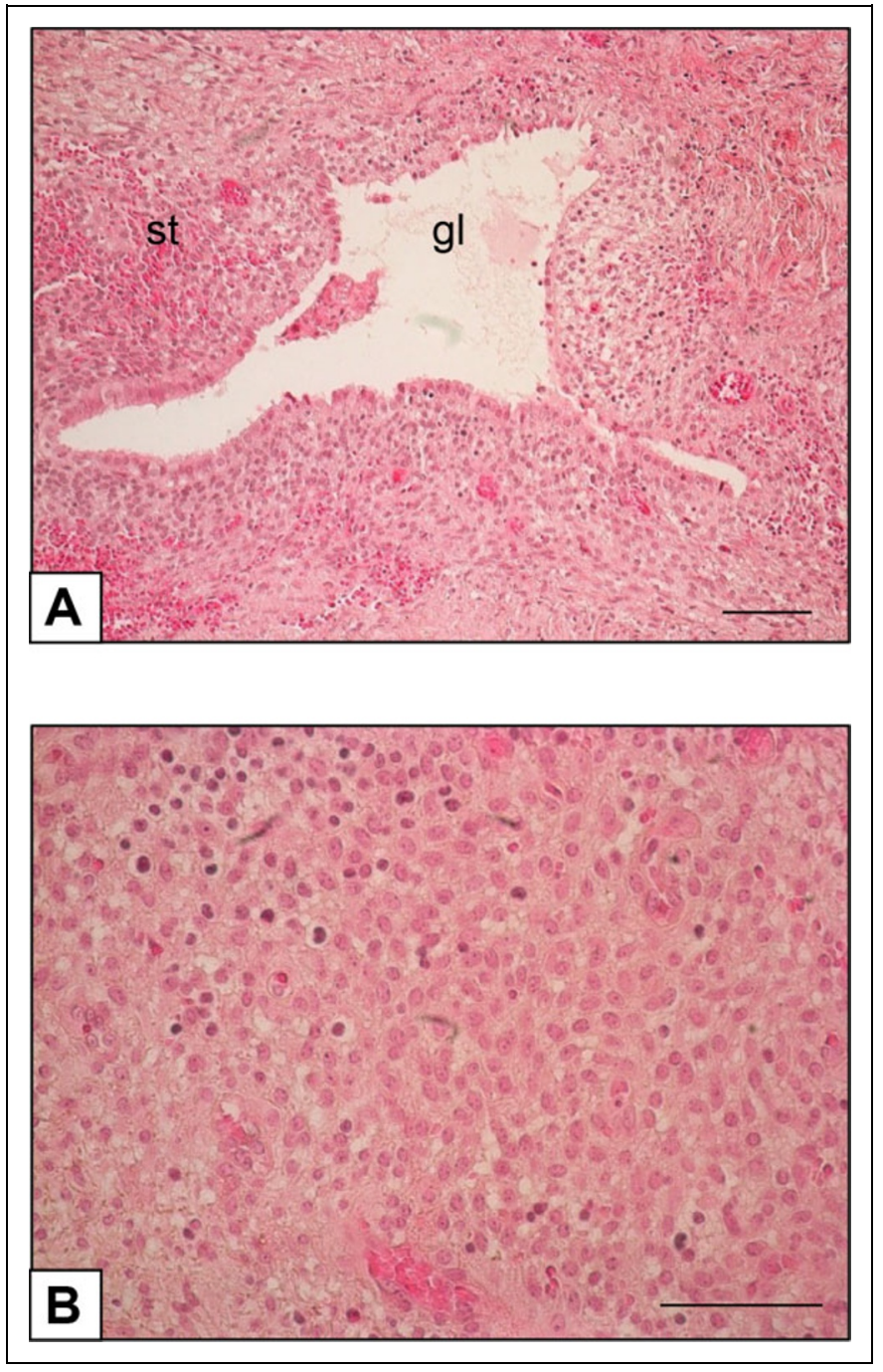

Figure I. Histopathological aspect of sections from the ectopic endometriotic tissue in right lung stained by hematoxylin and eosin (H\&E). Tissue area showing altered endometriotic gland surrounded by a stromal layer (A). Tissue section area of the lung implant (H\&E) showing an isolated cluster composed of elongated stromal-like with ovoid nuclei, containing sparse inflammatory cells (B). Scale bar: $100 \mu \mathrm{m}$.

Abundant-specific expression of CD10 antigen was observed in the gland surrounding stromal cells, while the gland lining epithelial cells were not stained (Figure 3B). Comparatively, nuclear expression of estrogen receptors and progesterone protein receptors was detected in both the gland lining epithelial cells and in cells from the surrounding stroma, where a lower staining was observed on epithelial cells (Figure 3C and D). Additionally, the endometriotic tissue showed immunoreactivity for Wilms tumor protein, WT1, in the nuclei of gland lining epithelial cells (Figure 3E). PAX8 proteins were moderately expressed in the stromal clusters (Figure 3F; Table 1). Of note, only $4 \%$ to $5 \%$ of the cell nuclei from the stromal component were immunoreactive, with the Ki-67 antibody indicating proliferative activity, while the gland lining cuboidal cells were not stained (Table 1). The immunohistochemical analysis of sections 

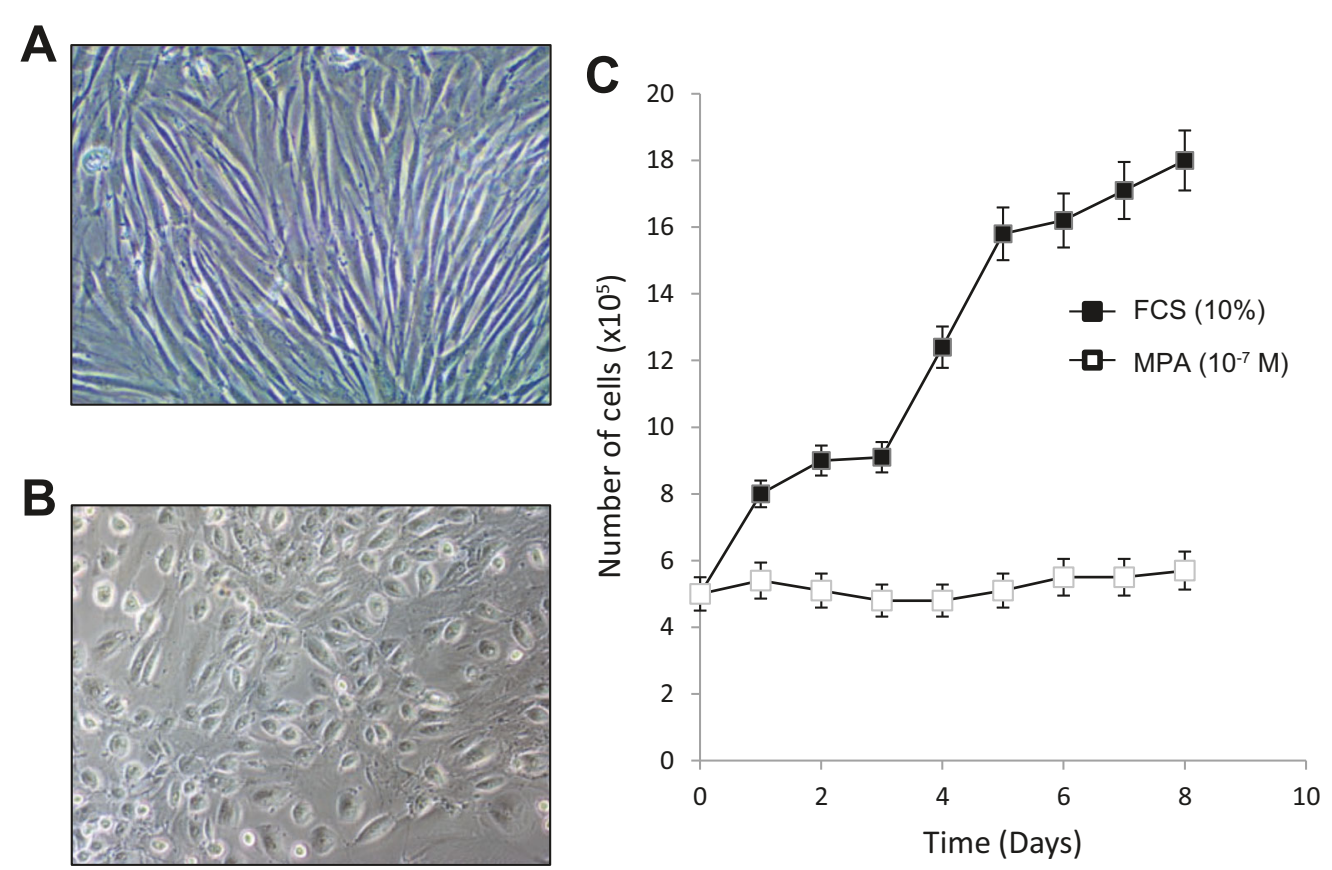

Figure 2. Phase-contrast microscopy and growth curve of TH-EMI cells. Spindle-shaped TH-EMI cells cultured in medium with $10 \%$ of fetal calf serum (FCS; A). Cultured TH-EMI cells treated with medroxyprogesterone acetate (MPA, $10^{-7} \mathrm{~mol} / \mathrm{L}$ ), showing appearance of large epitheloidlike cells (B). The cells in logarithmic growth phase seeded at $2 \times 10^{5} / \mathrm{mL}$ in medium with $10 \% \mathrm{FCS}$ and cells treated with $10^{-7} \mathrm{~mol} / \mathrm{L} \mathrm{MPA}$. The cell number of untreated (black squares) and MPA-treated cells (white squares) prepared in triplicate. Growth inhibition of the TH-EMI cells in a time course experiment (white squares; C). Each point represents the means \pm standard error (SE) of triplicate cultures evaluated in 2 separate experiments.

prepared from the diaphragmatic lesion showed comparable phenotypic profile (not shown).

The detailed immunocytochemical traits of TH-EM1 cell line are shown in Figure 4A to F and Table 1. While the cells exhibited intense membrane and cytoplasmic staining for the vimentin protein (Figure 4A), weak cytoplasmic immunolabeling for cytokeratin antibody was found in low percentage of the larger size TH-EM1 cells (Figure 4B; Table 1). Remarkably, intense immunoreactivity for the erythropoietin receptor was detected at the membrane in more than $70 \%$ of the cells (Figure $4 \mathrm{C}$ ), while $\mathrm{Fet}-\mathrm{Hb}$ was revealed in the cytoplasm of more than $60 \%$ of the cells (Figure 4D; Table 1). Consistent nuclear immunostaining for SOX-2 protein was present in $76 \%$ of the cells (Figure 4E; Table 1), Nanog in 49\%, and Oct3/4 in approximately $12 \%$ of the cells (Table 1 ). The nuclear expression of PAX8 was observed in 54\% of the TH-EM1 cells (Figure 4F; Table 1). The cells were weakly or not immunostained with anti-CD31, CD79, and anti-HLA-DR antibodies (Table 1).

\section{Flow Cytometry}

The flow cytometry analysis revealed that the unsorted adherent TH-EM1 cells from different passages were strongly immunoreactive for the mesenchymal stromal cell markers CD9, CD13, CD73, and CD90 (Figure 5A). In addition, the cells were consistently positive for CD105 (endoglin) expressed in
$74 \%$ and CD157 (BST1) protein expressed in $70 \%$ of the THEM1 cells (Figure 5A; Table 1). Also, the analysis revealed consistent expression of the hematopoietic markers, that is, the $\alpha$-globin molecule found in $65 \%$ of the cells and Fet-Hb found in $76 \%$ of the cells (Figure 5B; Table 1). Among the embryonic markers, the SOX-2 molecules were detected in $70 \%$ of the cells, while the Nanog molecules were detected in $52 \%$ of them (Figure 5B; Table 1). The Oct-3/4 embryonic marker molecules were detected in $15 \%$ of the cells (not shown).

\section{Cytogenetic and Microarray Chromosomal Findings}

The RGH obtained from dividing TH-EM1 cells examined at different in vitro passages (4th, 15th, and 43rd) demonstrated predominantly normal cellular metaphases (Figure 6A). Microarray chromosomal analysis performed with TH-EM1 cellular DNA isolated from the early passage (fourth) did not reveal histograms indicating significant unbalanced structural variations or aneuploidy. On the contrary, presence of distinctive gains of DNA sequences located at $8 \mathrm{p} 23.1,11 \mathrm{p} 15.5$, and 12p11.23 chromosomal segments were detected (Figure 6B).

\section{Discussion}

Previous culture experience of endometriosis-derived cells has been performed and often resulted in short-term cultures that 

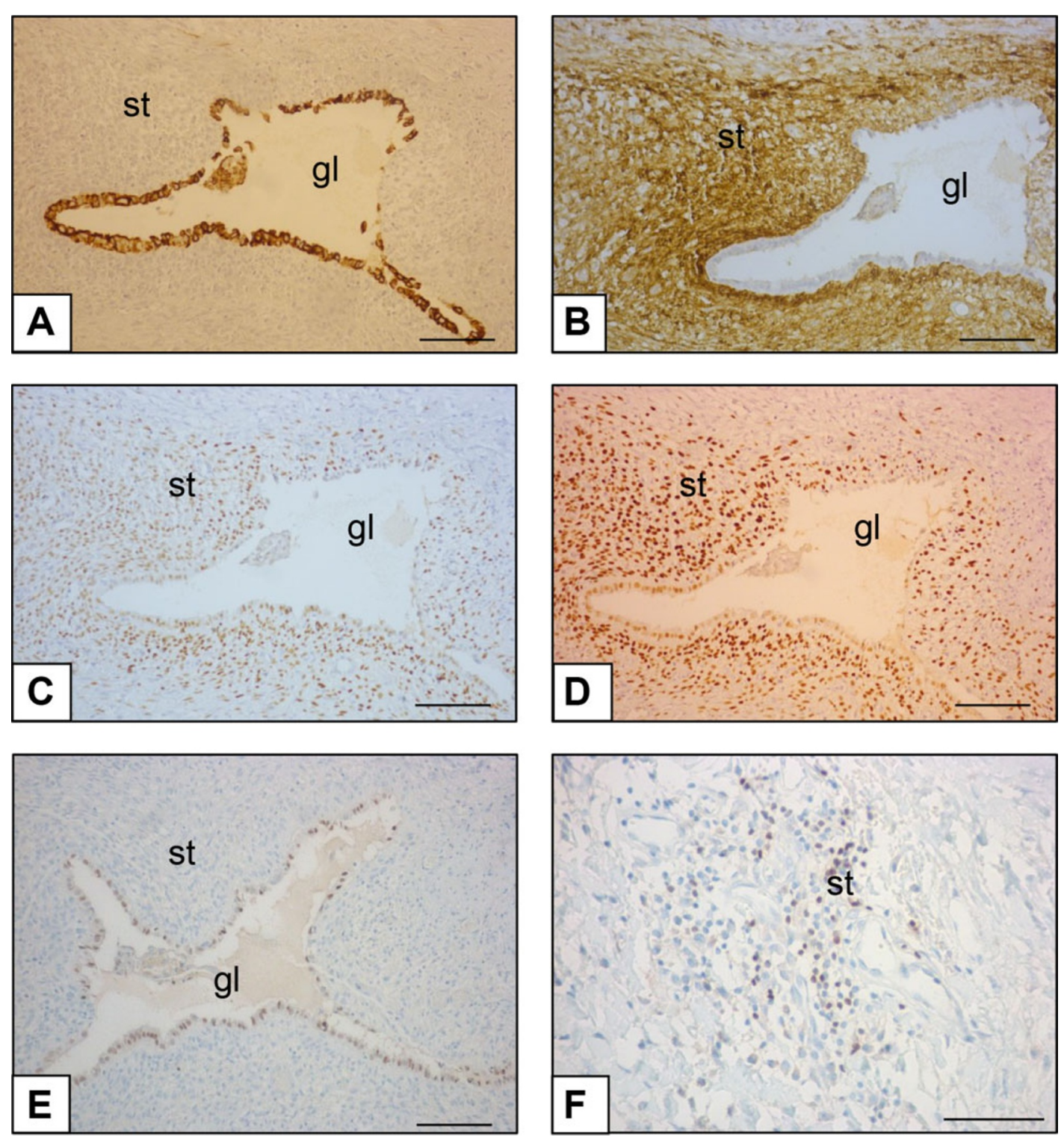

Figure 3. Representative immunostaining of a serial tissue sections of the endometriotic tissue in lung parenchyma with anticytokeratin (A); anti-CDIO (B); anti-estrogen receptor (ER; C); anti-progesterone receptor (PR; D); anti-WTI (E); and anti PAX8 antibodies. Note: The expression of PAX 8 molecules exclusively in cells from stromal clusters $(*)(F)$. Scale bar: $100 \mu \mathrm{m}$.

however allowed studies of cell morphology, aromatase activity, lipid metabolism, cytokine production, and endometriotic cell differentiation capacities. ${ }^{13,14}$ Although subsequent passages were sometimes achieved, such studies have strengthened the notion that both epithelial and stromal endometriotic cells have a limited life span in vitro. In this report, we describe the proliferative, phenotypic, and cytogenetic characteristics of a novel spontaneously growing TH-EM1 cell line derived from an ectopic endometriotic implant developed in lung tissue. Phenotypically, the TH-EM1 cells strongly express several mesenchymal stromal markers including CD9, CD13, CD73, CD90, CD105, and CD157. Conversely, they were not immunoreactive for CD31, CD79, and HLA-DR antigens. The mesenchymal cell type was further confirmed by the intense staining for vimentin and CD10 antigens and by the weak labeling of epithelial marker molecules. This is in agreement with a number of studies that have reported consistent expression of the CD73, CD90, C105, and CD157 molecules in mesenchymal stromal lineages established from the endometrium and the bone marrow tissues. ${ }^{10,15}$ Specifically, both the CD105 or endoglin and CD157 play an essential role in the development of endometriosis, where the expression of CD105 is related to the active angiogenesis, ${ }^{16}$ while CD157 is involved in the regulation of cell adhesion and migration processes. ${ }^{17}$

Remarkably, our immunocytochemical and flow cytometry analyses confirmed coexpression of the hematopoietic and embryonic markers in TH-EM1 cells that were also found in human mesenchymal stem cells derived from bone marrow, 

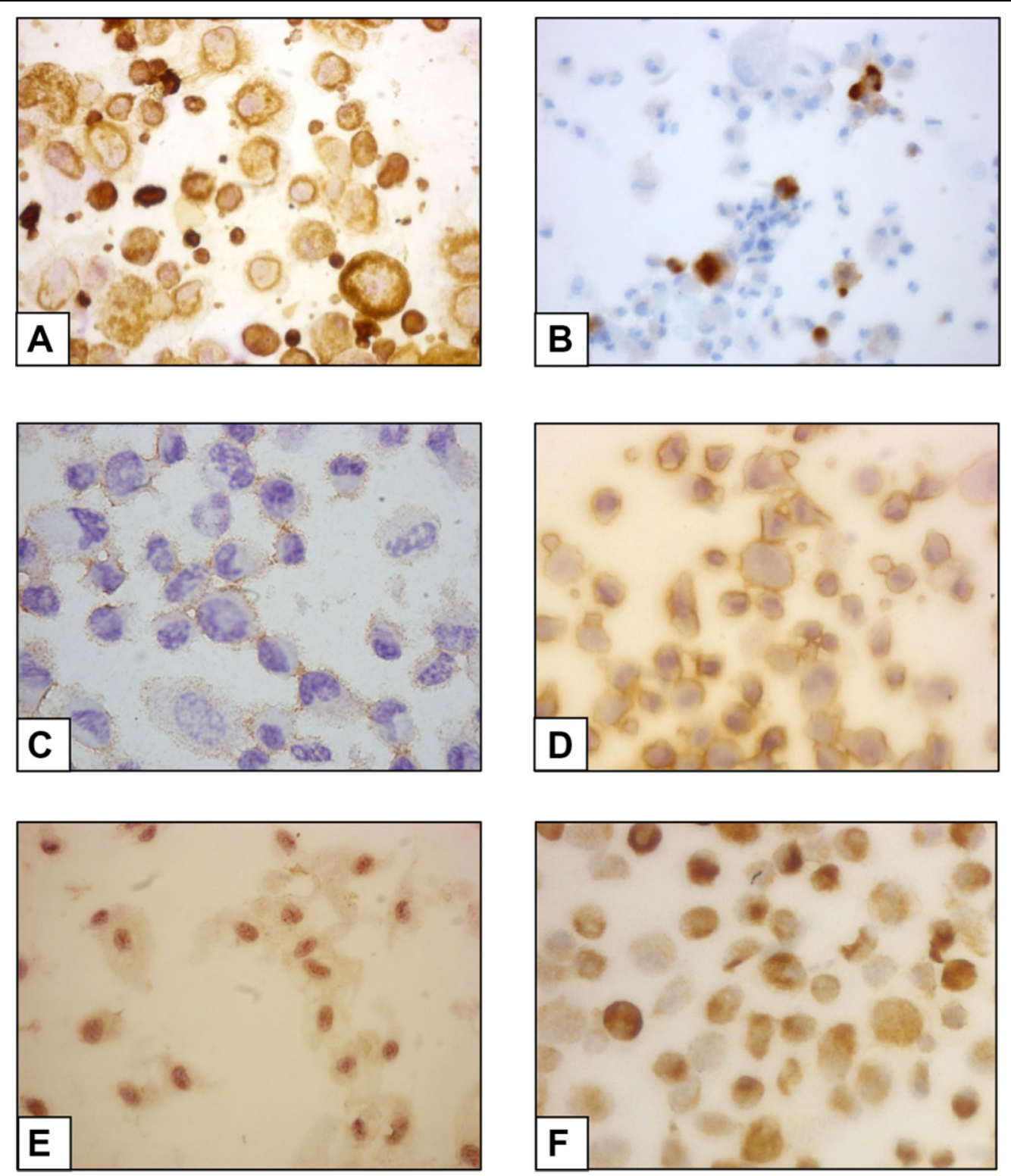

Figure 4. Representative immunostaining of TH-EMI cells prepared as cytospin smears and treated with anti-vimentin (A); anti-pan cytokeratin (B); anti-erythropoietin receptor (EpoR; C); anti-fetal hemoglobin (Fet-Hb; D); anti-SOX2 (E); and anti-PAX 8 antibodies (F).

adipose tissue, heart, and dermis. ${ }^{18}$ Of note, the expression of the erythropoietin receptors, $\alpha$-globin, and Fet-Hb markers suggests a stromal lineage expressing similar phenotypic markers to those observed in bone marrow mesenchymal stromal cells. ${ }^{19}$ In this regard, it has been demonstrated that in human bone marrow recipients, donor-derived bone marrow cells have the capacity to differentiate into uterine endometrium. ${ }^{20}$ The expression of the erythropoietin receptor and its ligand was earlier reported in both normal endometrium and endometriotic tissues, suggesting an autocrine growth control of endometriotic tissue. ${ }^{21,22}$

Concerning SOX-2, Nanog, and Oct3/4 embryonic markers, their expression has been specifically recognized in normal endometrium, endometriosis, and endometrial cancer tissues. ${ }^{23}$ In this respect, it was suggested that aberrant expression of SOX-2 is connected to a stem cell origin of endometriosis, whereas the expression of all progenitor markers would indicate that the endometrial tissue as a source of pluripotent stem cells. ${ }^{24}$ Likewise, the expression of SOX-2, Nanog, and Oct3/4 has been observed in mesenchymal stromal cells from fetal lung having the capacity to differentiate into cells from the 3 germ cell layers. ${ }^{25}$ In this regard, our preliminary results showed that treatment of TH-EM1 cells with adipogenic medium induced their differentiation into adipocytic cells as confirmed by the morphological changes and by Oil-red-O staining of cytoplasmic lipid droplets (not shown). 


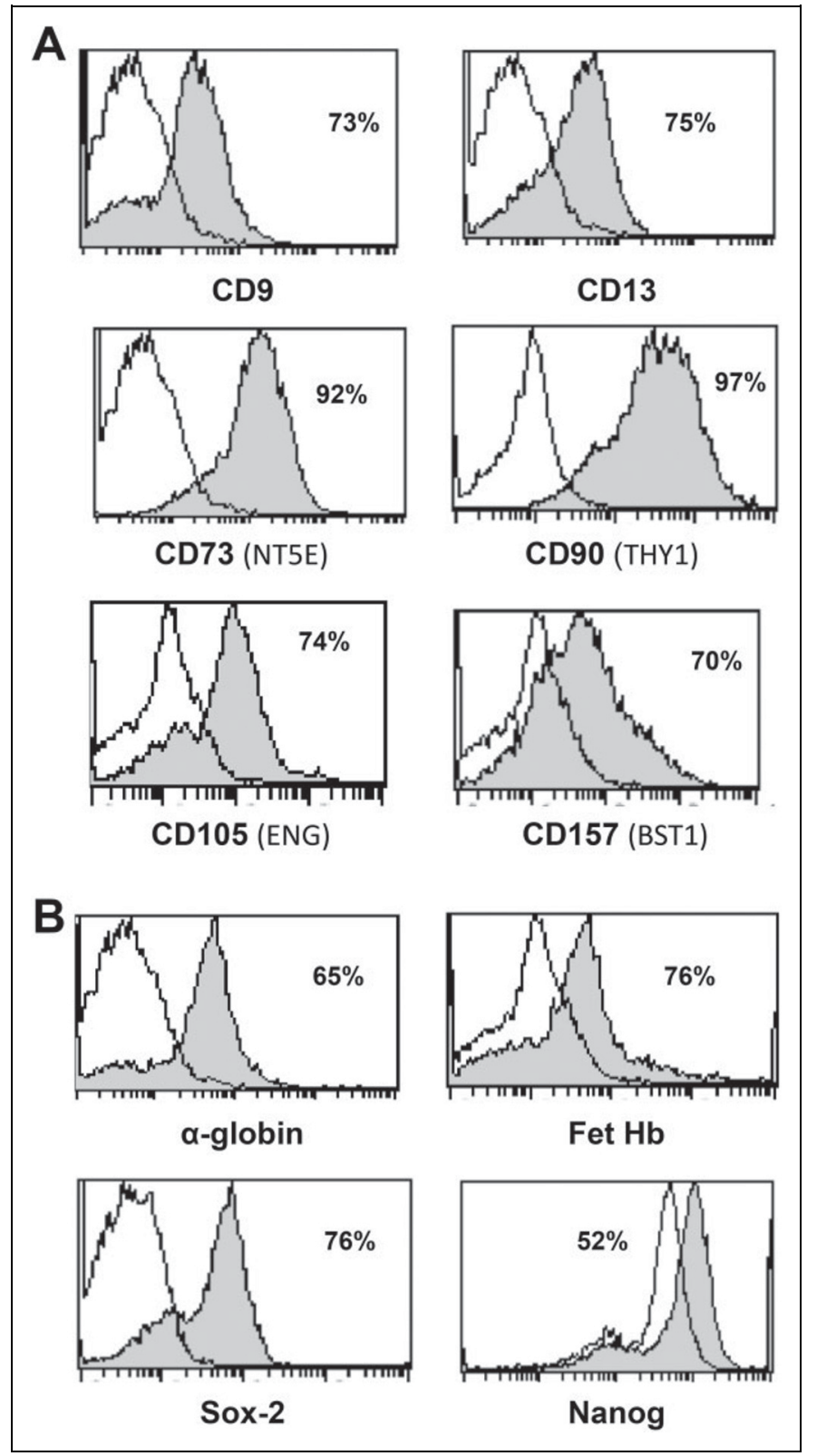

Figure 5. Flow cytometry analysis. Representative flow cytometry profiles were obtained after incubation of TH-EMI cells with different fluorescence-labeled antibodies. Level of expression of mesenchymal stromal cell markers: CD9, CDI3, CD73, CD90, CDI05 and CI57 (A), hematopoietic markers $\alpha$-globin, Fet- $\mathrm{Hb}$, and the embryonic markers SOX-2 and Nanog (B). Data are presented as fluorescenceactivated cell sorting graphs (mean fluorescence intensity [MFI]), where the dark curve shows fluorescence intensity of the marker antibody, while white curve area expresses background fluorescence intensity of control antibody.

In general, it is believed that pelvic endometriosis is a precursor lesion linked to the development of TE. ${ }^{2,7}$ However, it has been reported that concomitant thoracic and pelvic endometriosis is present in less than $18 \%$ in a cohort of 110 patients with TE that clinically contradicts the common migration possibility of endometriotic tissue. ${ }^{2}$ Accordingly, these studies suggested that absence of pelvic endometriosis in cases with TE strengthens the hypothesis that extrapelvic endometriosis may start as an independent pathological process. ${ }^{2,4,12}$ Moreover, it has been reported that only $25 \%$ of the cases with TE contain both the differentiated endometrial-like glands and endometriotic stromal tissue. ${ }^{26}$ Hence, it was supposed that the differentiation of stromal cells of pelvic origin is inhibited in the thoracic cavity environment since only sparse endometrial-like glands are observed. ${ }^{27}$ Another discrepancy with the migration theory might be, for example, the development of endometriotic tissue in the urinary system in men treated with estrogens for prostate cancer, suggesting existence of pluripotential cells in situ able to change into endometrial tissue. ${ }^{28,29} \mathrm{In}$ fact, de novo differentiation of nonendometrial mesenchymal stem cells might be a conceivable explanation for the development of misplaced endometriosis in skin, cerebellum, and lung. ${ }^{30-32}$ Regarding the histogenetic origin of the TE cell line in our case, both the phenotypic profile and the fact that the patient was previously treated for pelvic endometriosis were conceivable that the cells developed from a clone interrelated to an endometrium-derived mesenchymal stroma cell category. For comparison, the revealed $\mathrm{CD} 9, \mathrm{CD} 13, \mathrm{CD} 73, \mathrm{CD} 90$, and $\mathrm{CD} 105$ cell markers in TH-EM1 cell line were also observed in both immortalized endometrial stromal cells and endometrial mesenchymal stem cells established from lesions and eutopic endometrium. ${ }^{10,33,34}$ However, the coexpression of hematopoietic and embryonic markers observed in TH-EM1 cells are not yet described in endometriosis-related stromal cell line.

However, although the phenotype of TH-EM1 cell lineage suggests a clone related to a multipotent mesenchymal stroma from endometrial origin, the uncommon expression of hematopoietic markers remind a cell clone phenotypically related to bone marrow stem cells likewise implicated in TE pathogenesis. ${ }^{35,36}$ Nonetheless, because of the great morphological and cytochemical variability in mesenchymal stromal cells derived from the endometrium, placenta, skin, bone, or adipose tissue, ${ }^{37-39}$ it is at present difficult to firmly ascertain the accurate origin of the TH-EM1 cell lineage.

Finally, specific gains of DNA sequences were revealed on the chromosomal segments $8 \mathrm{p} 23.1,11 \mathrm{p} 15.5$, and $12 \mathrm{p} 11.23$ by the microarray analysis. Although copy number variations of the genes located at the amplified segments have been described in the database of genomic variants of general population, ${ }^{40}$ further studies are necessary to define whether some of these amplifications are specifically involved in the pathogenesis of TE syndrome.

In conclusion, the TE-derived TH-EM1 cell line, stably retaining features of immature stromal derived cell type, appears useful for multidisciplinary studies such as cell marker profiling, decidualization process, and biological behavior after stimulation. The consistent coexpression of embryonic and hematopoietic markers is a novel finding that may contribute to better define the phenotype, differentiation capacities, and the functional properties of stromal cells constitutive of misplaced endometriotic tissues. Finally, the molecular marker profile and the genomic distinctiveness of the TH-EM1 cell 


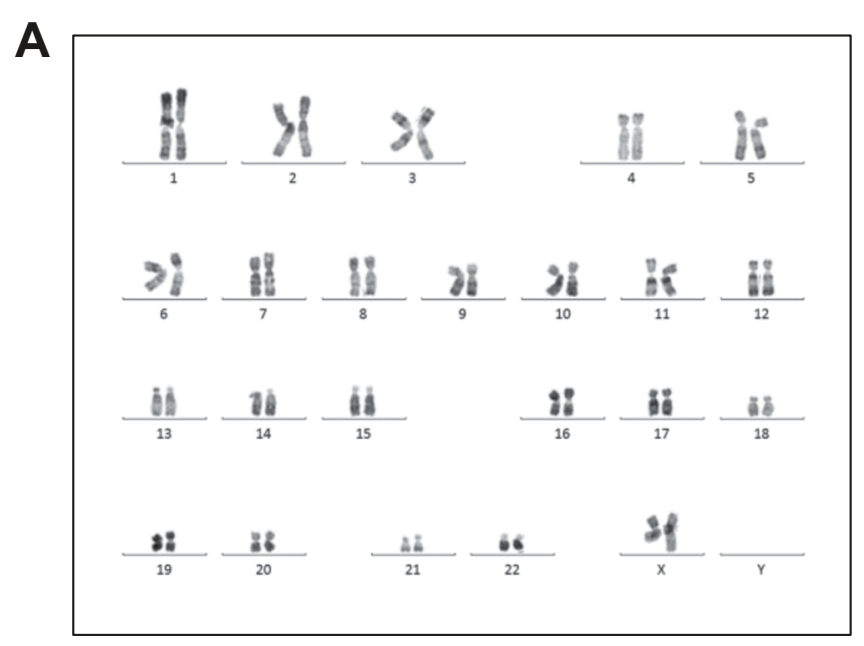

B

\begin{tabular}{|l|l|l|l|l|}
\hline $\begin{array}{c}\text { Copy } \\
\text { Number } \\
\text { variation }\end{array}$ & \multicolumn{1}{|c|}{ Location (GRCh37/hg19) } & Cytoband & Size(kb) & \multicolumn{1}{|c|}{ OMIM Genes* } \\
\hline Gain & Chr8: 7053186-8079920 & $p 23.1$ & 1,027 & DEFB103A and DEFB4A \\
\hline Gain & Chr11: 67374414-67828350 & $q 13.2$ & 454 & $\begin{array}{l}\text { ALDH3B2, ACY3, } \\
\text { NDUFS8, CHKA, } \\
\text { UNC93B1, TBX10, } \\
\text { ALDH3B1, TCIRG1 and } \\
\text { NDUFV1 }\end{array}$ \\
\hline Gain & Chr12: 27306320-27760152 & $\mathrm{p} 11.23$ & 454 & STK38L and ARNTL2 \\
\hline
\end{tabular}

*http:/www.ncbi.nlm.nih.gov/omim/

Figure 6. Conventional cytogenetic and microarray chromosomal analysis. TH-EMI cells metaphase spread (RHG-banding) from the fourth passage showing normal karyotype $(46 \mathrm{XX})(\mathrm{A})$. The microarray chromosome analysis (MCA) performed with TH-EMI cellular DNA hybridized on Agilent array. Distinct gains of DNA sequences located at 8p23.I, IIpI5.5, and I2p II.23 chromosomal segments are observed (B).

line might delineate novel molecular pathways in TE syndrome development and progression in women.

\section{Acknowledgements}

The authors are indebted to Dr Celine Méhats for the invaluable help during the manuscript writing and revision.

\section{Declaration of Conflicting Interests}

The author(s) declared no potential conflicts of interest with respect to the research, authorship, and/or publication of this article.

\section{Funding}

The author(s) received no financial support for the research, authorship, and/or publication of this article.

\section{References}

1. Alifano M. Catamenial pneumothorax. Curr Opin Pulm Med. 2010;16(4):381-386.

2. Channabasavaiah AD, Joseph JV. Thoracic endometriosis: revisiting the association between clinical presentation and thoracic pathology based on thoracoscopic findings in 110 patients. Medicine (Baltimore). 2010;89(3):183-188.

3. Honore GM. Extrapelvic endometriosis. Clin Obstet Gynecol. 1999;42(3):699-711.

4. Nair SS, Nayar J. Thoracic endometriosis syndrome: a veritable Pandora's box. J Clin Diagn Res. 2016;10(4):QR04-QR08.

5. Joseph J, Sahn SA. Thoracic endometriosis syndrome: new observations from an analysis of 110 cases. Am J Med. 1996;100(2): 164-170.

6. Augoulea A, Lambrinoudaki I, Christodoulakos G. Thoracic endometriosis syndrome. Respiration. 2008;75(1):113-119.

7. Soriano D, Schonman R, Gat I, et al. Thoracic endometriosis syndrome is strongly associated with severe pelvic endometriosis and infertility. J Minim Invasive Gynecol. 2012;19(6): 742-748.

8. Markham SM, Carpenter SE, Rock JA. Extrapelvic endometriosis. Obstet Gynecol Clin North Am. 1989;16(1):193-219.

9. Korom S, Canyurt H, Missbach A, et al. Catamenial pneumothorax revisited: clinical approach and systematic review of the literature. J Thorac Cardiovasc Surg. 2004;128(4):502-508. 
10. Li F, Alderman MH 3 rd, Tal A, et al. Hematogenous dissemination of mesenchymal stem cells from endometriosis. Stem Cells. 2018;36(6):881-890.

11. Maruyama T, Yoshimura Y. Stem cell theory for the pathogenesis of endometriosis. Front Biosci (Elite Ed). 2012;4:2754-2763.

12. Suginami H. A reappraisal of the coelomic metaplasia theory by reviewing endometriosis occurring in unusual sites and instances. Am J Obstet Gynecol. 1991;165(1):214-218.

13. Bulun SE, Monsivais D, Kakinuma T, et al. Molecular biology of endometriosis: from aromatase to genomic abnormalities. Semin Reprod Med. 2015;33(3):220-224.

14. Giudice LC.Clinical practice. Endometriosis. $N$ Engl J Med. 2010;362(25):2389-2398.

15. Jiang Y, Jahagirdar BN, Reinhardt RL, et al. Pluripotency of mesenchymal stem cells derived from adult marrow. Nature. 2002;418(6893):41-49.

16. Hayrabedyan S, Kyurkchiev S, Kehayov I. Endoglin (cd105) and S100A13 as markers of active angiogenesis in endometriosis. Reprod Biol. 2005;5(1):51-67.

17. Ortolan E, Arisio R, Morone S, et al. Functional role and prognostic significance of CD157 in ovarian carcinoma. J Natl Cancer Inst. 2010;102(15):1160-1177.

18. Riekstina U, Cakstina I, Parfejevs V, et al. Embryonic stem cell marker expression pattern in human mesenchymal stem cells derived from bone marrow, adipose tissue, heart and dermis. Stem Cell Rev. 2009;5(4):378-386.

19. Alcayaga-Miranda F, Cuenca J, Luz-Crawford P, et al. Characterization of menstrual stem cells: angiogenic effect, migration and hematopoietic stem cell support in comparison with bone marrow mesenchymal stem cells. Stem Cell Res Ther. 2015;6:32.

20. Taylor HS. Endometrial cells derived from donor stem cells in bone marrow transplant recipients. JAMA. 2004;292(1):81-85.

21. Matsuzaki S, Canis M, Yokomizo R, Yaegashi N, Bruhat MA, Okamura K. Expression of erythropoietin and erythropoietin receptor in peritoneal endometriosis. Hum Reprod. 2003;18(1): 152-156.

22. Yokomizo R, Matsuzaki S, Uehara S, Murakami T, Yaegashi N, Okamura K. Erythropoietin and erythropoietin receptor expression in human endometrium throughout the menstrual cycle. Mol Hum Reprod. 2002;8(5):441-446.

23. Song Y, Xiao L, Fu J, et al. Increased expression of the pluripotency markers sex-determining region Y-box 2 and Nanog homeobox in ovarian endometriosis. Reprod Biol Endocrinol. 2014;12:42.

24. Gotte M, Wolf M, Staebler A, Buchweitz O, Kiesel L, Schüring AN. Aberrant expression of the pluripotency marker SOX-2 in endometriosis. Fertil Steril. 2011;95(1):338-341.

25. Zheng C, Yang S, Guo Z, et al. Human multipotent mesenchymal stromal cells from fetal lung expressing pluripotent markers and differentiating into cell types of three germ layers. Cell Transplant. 2009;18(10):1093-1109.

26. Haga T, Kurihara M, Kataoka H, Ebana H. Clinical-pathological findings of catamenial pneumothorax: comparison between recurrent cases and non-recurrent cases. Ann Thorac Cardiovasc Surg. 2014;20(3):202-206.

27. Haga T, Kumasaka T, Kurihara M, Kataoka H, Miura M. Immunohistochemical analysis of thoracic endometriosis. Pathol Int. 2013;63(9):429-434.

28. Martin JD, Jr., Hauck AE. Endometriosis in the male. Am Surg. 1985;51(7):426-430.

29. Pinkert TC, Catlow CE, Straus R. Endometriosis of the urinary bladder in a man with prostatic carcinoma. Cancer. 1979;43(4): 1562-1567.

30. Hobbs JE, Bortnick AR. Endometriosis of the Lungs. Am J Obstetric Gynecol. 1940;40(5):832-843.

31. Sarma D, Iyengar P, Marotta TR, terBrugge KG, Gentili F, Halliday W. Cerebellar endometriosis. AJR Am J Roentgenol. 2004; 182(6):1543-1546.

32. von Stemm AM, Meigel WN, Scheidel P, Gocht A. Umbilical endometriosis. J Eur Acad Dermatol Venereol. 1999;12(1):30-32.

33. Cheng Y, Li L, Wang D, et al. Characteristics of human endometrium-derived mesenchymal stem cells and their tropism to endometriosis. Stem Cells Int. 2017;2017:4794827.

34. Gargett CE, Schwab KE, Deane JA. Endometrial stem/progenitor cells: the first 10 years. Hum Reprod Update. 2016;22(2): 137-163.

35. Rolfing JH, Baatrup A, Stiehler M, Jensen J, Lysdahl H, Bünger C. The osteogenic effect of erythropoietin on human mesenchymal stromal cells is dose-dependent and involves nonhematopoietic receptors and multiple intracellular signaling pathways. Stem Cell Rev. 2014;10(1):69-78.

36. Zwezdaryk KJ, Coffelt SB, Figueroa YG, et al. Erythropoietin, a hypoxia-regulated factor, elicits a pro-angiogenic program in human mesenchymal stem cells. Exp Hematol. 2007;35(4): 640-652.

37. Buhring HJ, Battula VL, Treml S, Schewe B, Kanz L, Vogel W. Novel markers for the prospective isolation of human MSC. Ann N Y Acad Sci. 2007;1106:262-271.

38. Dominici M, Le Blanc K, Mueller I, et al. Minimal criteria for defining multipotent mesenchymal stromal cells. The International Society for Cellular Therapy position statement. Cytotherapy. 2006;8(4):315-317.

39. Le Blanc K, Davies LC. MSCs-cells with many sides. Cytotherapy. 2018;20(3):273-278.

40. MacDonald JR, Ziman R, Yuen RK, Feuk L, Scherer SW. The database of genomic variants: a curated collection of structural variation in the human genome. Nucleic Acids Res. 2014;42(Database issue):D986-D992. 